\title{
During COVID-19 Pandemic Anesthesia Practices and Operating Room Management of Our Anesthesiology and Reanimation Clinic in the University of Health Sciences Turkey, Prof. Dr. Cemil Taşcıoğlu City Hospital
}

\author{
(D) Serap Karacalar, (D) Esra Aktaş Tekin, (D) Onur Okur, iD ibrahim Gün, (D) Tarkan Mıngır \\ University of Health Sciences Turkey, Prof. Dr. Cemil Taşcıoğlu City Hospital, Clinic of Anaesthesiology, Istanbul, Turkey
}

\section{Abstract}

Coronavirus Disease-2019 (COVID-19), which emerged in China in December 2019, was announced by the World Health Organization as a pandemic in March 2020. Many institutions and organizations in the world and Turkey have made recommendations on measures that will protect both medical personnel and the patient in cases requiring emergency or elective surgery in patients with suspected or confirmed COVID-19 and are still continuing to update their recommendations. In this article, we planned to convey our experiences regarding anesthesia and operating room practices that we carried out in line with the recommendations of the Ministry of Health Science Committee and other important health institutions in the world during this extraordinary period we passed.

Keywords: COVID-19, coronavirus, anesthesia, operating room, personal protective equipment, pandemic

\section{INTRODUCTION}

The world became aware of the Coronavirus Disease-2019 (COVID-19) outbreak for the first time when China reported to the World Health Organization (WHO) that patients with pneumonia of unknown origin occurred in the city of Wuhan, Hubey province on December 31, 2019. A process that had not been experienced before for the whole world began when the outbreak, which was perceived as a regional epidemic, initially affecting China and its surroundings, rapidly crossed Asian lands and began to threaten public health globally. In this period, we started our work in our hospital, of which name was Okmeydanı Education and Research Hospital before the pandemic and which was named as Prof. Dr. emil Taşçıoğlu City Hospital after the precious lecturer of İstanbul University Faculty of Medicine lost his life in the pandemic, primarily by holding informative meetings for the staff of our clinic and other employees in the operating room. In these meetings, we shared up-to-date information on transmission routes and prevention methods. Each meeting was held repeatedly at different times so that all personnel could attend. In the continuation of the process, we have completed our preparations for protection plannings that develop when needed. In this review, we have planned to transfer our experiences regarding our anesthesia and operating room practices during the COVID-19 pandemic process by dividing the process into three periods from the beginning of the pandemic. Because we think that since the announcement of "International Public Health Emergency" by WHO on January 30, three separate periods, which are different from each other in terms of dynamics, have been passed both in our own hospital and other regional hospitals. We have described these three periods as the first period from the announcement of the epidemic in China to the announcement 
of pandemic, the second period we have called the pandemic period and the third period when the normalization process has started.

\section{Our Anesthesia Practices and Operating Room Management During the COVID-19 Pandemic Process}

\section{Period I (December 2019 - March 11, 2020)}

In this process, from the endemic announcement in China to the pandemic announcement, which we considered as the first period, we tried to have the most detailed and accurate information about the general risks that might be encountered in these patients by evaluating the literature available for that period, mostly from China. Our aim was to make the right arrangements in order to protect all operating room health personnel and other patients from contamination, while providing the necessary treatment to the patients in line with their needs in case the pandemic started to rise in our country and istanbul and the number of patients increased. In this period, we could make the training meetings more frequently and we could rarely made them in the period II and III due to the workload and protective measures by providing the social distance and protection rules. Starting most of the trainings in the first period provided a very positive contribution to the system as it provided the basis for the team to protect itself and its surroundings in future periods which would be very busy. In the first period of the epidemic, all elective interventions in and out of the operating room continued routinely in our hospital, as in other hospitals. Since there was no patient volume outside of working hours during this period, the healthcare personnel in our clinic continued their normal working hours. In addition, we completed our workforce and equipment planning in case of an increase in patient volume during this period. We thought that this first period was a preparation period for pandemic for our hospital and clinic and this preparation period contributed a lot to the successful management of the pandemic.

\section{Period II: The Pandemic Period (March 11, 2020 - June 1, 2020)}

Along with the determination of the first patient in our country and the announcement of the pandemic, a Scientific Board was created for COVID-19 in many medical institutions and our hospital, especially the Ministry of Health. As the Anesthesiology and Reanimation Clinic, we determined the personal protection equipment (PPE) (N95/FFP2 or N99/FFP3 mask, waterproof apron, coveralls, gloves, bonnets, boots, and galoshes) which were required to protect the health of all employees who would support the treatment of patients with COVID-19 and prevent contamination. Then we conveyed the missing ones to the hospital administration department (Figure 1) (1). During this extraordinary period, we observed that close contact of the field with hospital administrations was necessity in order to meet urgent needs quickly. During this period, we experienced that the complete provision of these equipment, which we determined for the safety of medical personnel and patients, made a very positive contribution to the morale and motivation of our working staff by making them feel safe. Since it is important to learn how to put on and remove these materials as well as procuring PPE in order to prevent contamination, and since contamination is reported especially during removal, we have given priority to giving recurrent simulation training to all personnel in the operating room on putting on and removing PPE (Figure 2) (1,2). During these trainings, we have paid special attention to the participants being in small groups, maintaining social distance and being masked. For PPE putting on and removing, we have made visual videos filmed in our own clinic to be shared on the common social networks of our clinic's health workers. In March 2020, the Science Board of the Ministry of Health recommended that planned surgeries and endoscopic interventions should not be performed during this period due to the increased perioperative risks in patients undergoing surgery, even if they were asymptomatic and to ensure the controlled consumption of resources. These suggestions were discussed

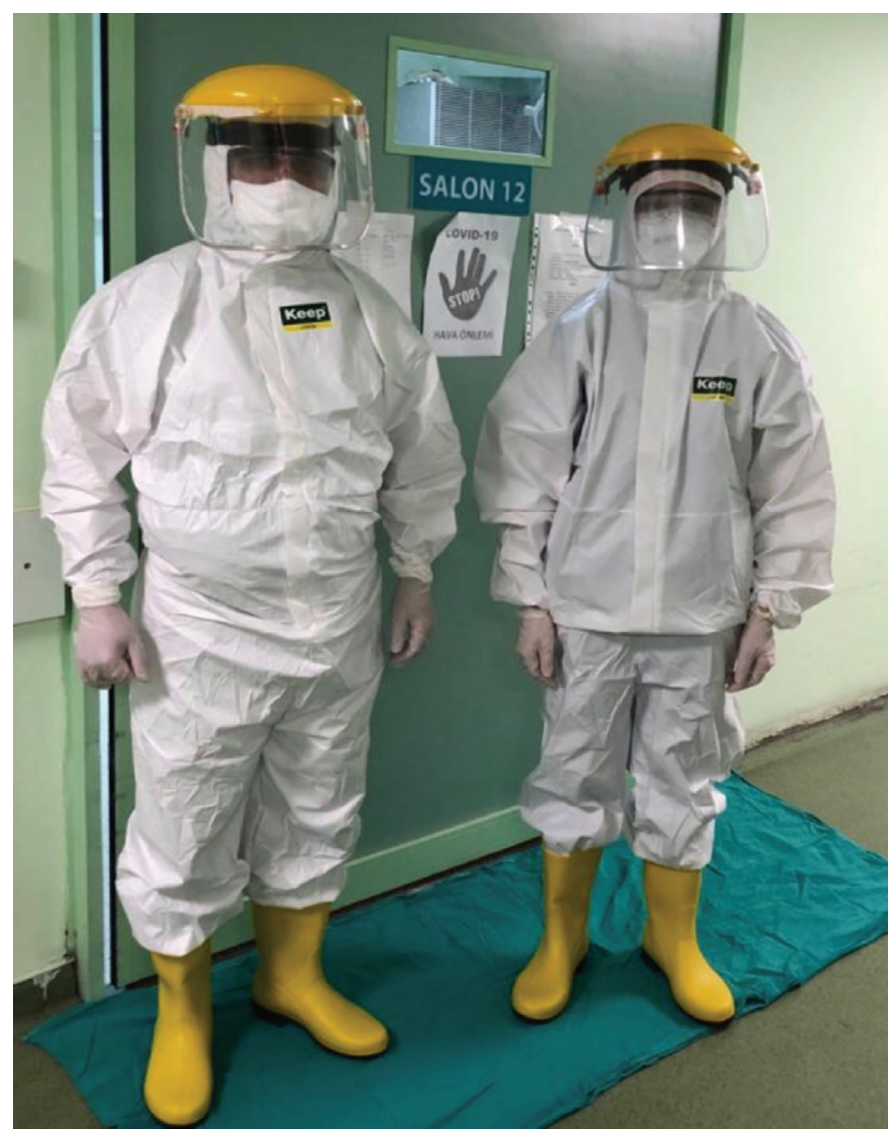

Figure 1. Personal protection equipment 
in the Scientific Committee of our hospital and as of March 21, elective surgeries and endoscopic interventions were postponed. Operations classified as urgent or emergent by various surgery societies including emergency cesarean section, acute abdomen, bleeding, orthopedic emergencies, large vessel emergencies, emergency bypasses, neurosurgeries and transplantations that could not be postponed were performed (3). In this period before the transition to normalization, all urgent surgeries of patients with and without COVID-19 were continued uninterruptedly in our hospital. In this period, while making the operation order

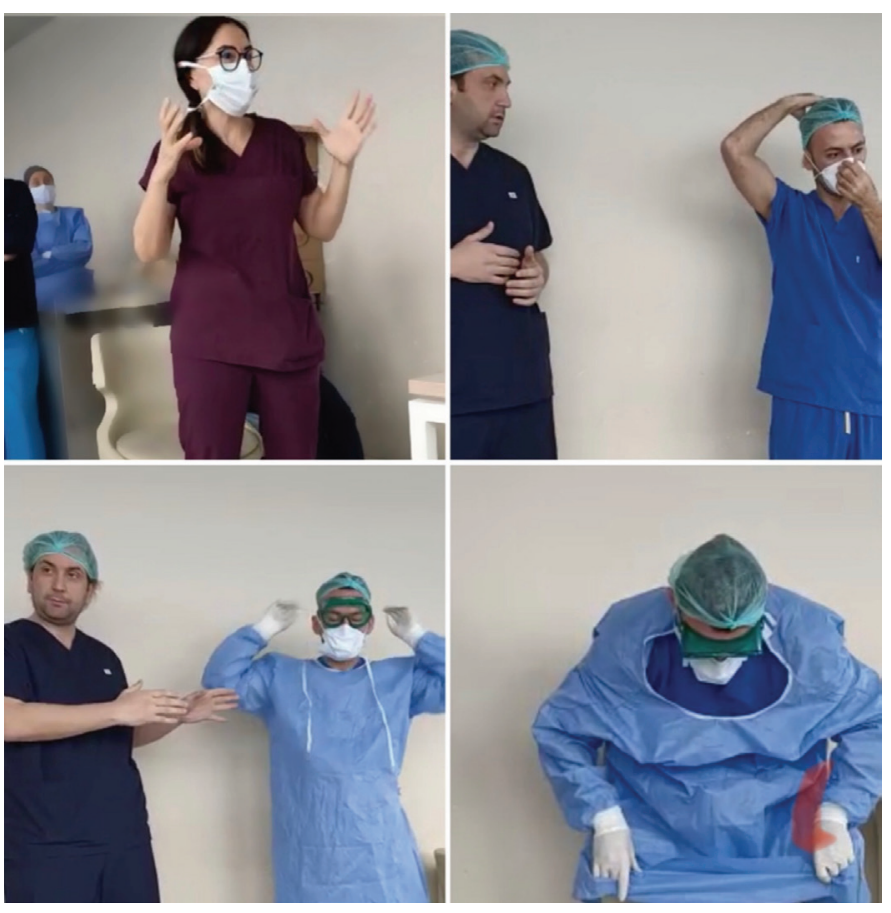

Figure 2. Simulation trainings of emergent patients, care was taken to start with patients with lower risk of operation, under 60 years of age and without comorbidity, in line with the recommendations (4-7).

\section{Period III: Normalization Process (June 1, 2020 - )}

Following the publication of the Scientific Committee of the Ministry of Health on a June 2020 "Working Guideline in Health Institutions During the Normalization Period", the process of returning to normal throughout our country has started. During this period, we started to gradually withdraw our human resources, which we had previously directed to our intensive care units, back to the operating room due to the increased surgical procedures. During this period, we did all plannings in our clinic, within the scope of the requests and the possibilities we had, by following the recommendations of the Ministry of Health's Scientific Board's current guides. The "Working Guideline in Health Institutions During the Normalization Period", which was last updated on July 7, 2020, emphasizes a planning to manage the increasing demand due to the postponed planned procedures in this process and ensuring the safety of patients and healthcare workers. Also it is emphasized that the COVID-19 related measures should be continued within this planning (8). In the same guideline, it is stated that it is necessary to observe a steady decrease in the incidence of COVID-19 for at least the last 14 days in order to start healthcare services to be provided especially to planned surgical patients at the provincial level, and it is appropriate to prioritize the operations by evaluating the patient, disease and surgical procedure factors when starting elective surgeries (Table 1) (8). In the current guideline, it is also recommended to establish a committee

Table 1. Factors belonging to the patient, disease and surgical procedure that should be considered in the prioritization of the operations in the normalization period

\begin{tabular}{|c|c|}
\hline Patient & $\begin{array}{l}\text { - Age } \\
\text { - Chronic diseases (COPD, asthma, CVD, CAD, malignancy, DM) } \\
\text { - Immunsupression (chemotherapy, immunosuppressive therapy for other reasons) } \\
\text { - Presence of COVID-19 or flu-like symptoms } \\
\text { - History of contact with a patient with COVID-19 in the last } 14 \text { days }\end{array}$ \\
\hline Disease & $\begin{array}{l}\text { - Whether there is a non-surgical treatment option } \\
\text { - The effect of delayed surgery on disease progression } \\
\text { - Whether the delay of the surgery will cause difficulty in the surgical technique }\end{array}$ \\
\hline Surgical procedure & $\begin{array}{l}\text { - Operation time } \\
\text { - Possibility of intubation } \\
\text { - Risk of the surgical area (head, neck, nose-throat, respiratory tract, thoracic surgery, etc.) } \\
\text { - The amount of blood loss predicted during surgery } \\
\text { - Number of people in the surgical team } \\
\text { - The necessity of postoperative intensive care follow-up } \\
\text { - Postoperative anticipated length of stay }\end{array}$ \\
\hline
\end{tabular}


consisting of administrative directors and representatives of infectious diseases, surgical departments, anesthesia, pathology, gastroenterology and other necessary disciplines in order to determine the planned surgical procedures to be performed in the hospital and to determine the priority surgical procedures to be performed for each unit by this committee (8).

\section{Workforce Planning in Our Anesthesia and Reanimation Clinic During the Pandemic Period}

In our clinic, especially with the announcement of the pandemic and the decision to cancel the elective surgeries, the patient load in the operating room decreased relatively, so our anesthesiologists, residents and technicians were divided into groups and included in shift-based working programs in order to employ the least number of personnel required. During this period, the patient load of our intensive care units was too high, so the working programs of our anesthesiologists, residents and technicians were mainly arranged to spend their shift in our intensive care units. In this period, the introduction of a flexible working system for all healthcare workers made it easier for us to plan working shift. In the first periods when the number of patients started to increase, the shifts continuing with 24-hour active 48-hour rest (24/48) periods were changed and reorganized as 24-hour active 72-hour rest (24/72) shifts, as it was stated that they were too consumer to be tolerated according to the feedback. During this period, all operating room employees were asked to evaluate themselves every day in terms of COVID-19 symptoms and contact history and to inform if they were symptomatic.

\section{Preoperative Evaluation of Patients Planned to be Operated During the Pandemic Period}

Patients with confirmed or suspected COVID-19 were identified before surgery and it was deemed appropriate to be taken into operation after two preoperative triages were made in line with the decisions of our hospital's Pandemic Scientific Committee in order to protect the safety of both themselves and healthcare professionals. The first and second triages are continued as follows.

First Triage: An anamnesis is taken by the relevant surgical service physician, and after physical examination (PE), personalized tests are requested. In terms of symptoms of COVID-19 (fever, cough, breathlessness, weakness, diarrhea, abdominal pain, muscle pain, sore throat, vomiting, loss of sense of taste and smell, etc.), the patient, and the people living in the same house and working in the same workplace with the patient and person who will accompany the patient in the hospital are questioned for the last 14 days, in terms of symptoms of COVID-19 (fever, cough, breathlessness, weakness, diarrhea, abdominal pain, muscle pain, sore throat, vomiting, loss of sense of taste and smell, etc.) (1). At the end of the evaluations, elective surgeries of the patients with suspected COVID-19 are canceled and they are referred to infectious diseases. If it is necessary to test for COVID-19 in line with the recommendations of the scientific board guide of the Ministry of Health, the sample is taken by the unit to which the patient is admitted. Until the test result is obtained, the patient is informed about isolation at home. The interventional procedure should be planned within three days at the latest after a negative test result (8).

In the current guideline, the conditions that require polymerase chain reaction (PCR) testing as soon as possible (48 hours) before the planned treatment or procedure are stated as follows;

- Patients with suspected COVID-19 or the contacts of these patients,

- If the frequency of new patients in the last 14 days (total number of new patients/population at risk in the last 14 days) is above $2 \%$, patients who are asymptomatic and even if there is no suspicious contact.

Regardless of the frequency of new cases in the last 14 days;

- Patients who have undergone chemotherapy and radiotherapy, solid organ transplantation, or bone marrow transplantation

- Patients using >15 mg prednisolone or equivalent glucocorticoid and/or small molecule or biological agent with immunosuppressive effect in the last three months,

- Group A surgeries of all surgical branches,

- Patients of Otorhinolaryngology and Plastic Surgery departments requiring head and neck surgery,

- All patients of Cardiac Surgery Deparment requiring access to the cardiopulmonary pump,

- Patients requiring thoracic surgery,

- Patients scheduled for jaw surgery,

- Patients scheduled for airway surgery,

- Patients scheduled for cancer surgery,

- All patients who will undergo transplantation,

- Surgical patients with an American Society of Anesthesiologists score of III or above and requiring general anesthesia.

If the first test result is negative but the suspicion of COVID-19 is high, it is recommended to perform the second test at least 24 hours later. If the incidence of new patients in the last 14 
days is below $2 \%$, testing is not recommended in patients who are asymptomatic and have no suspicious contact, except for the above-mentioned conditions.

The patient with a positive test result is managed according to the Ministry of Health COVID-19 Guidelines. Even if the test result is negative, N95/FFP2 mask, goggles/face protector should be used in processes that generate aerosol (1). Planned surgery should be postponed if the patient has positive test and/or symptoms compatible with COVID-19. The procedure of the patient should be performed as soon as possible (on the same day, if possible) after hospitalization, and the pre- and post-procedure periods should be kept short (8). The second triage of the patients is performed by the anesthesiologist before the intervention.

Second Triage: After anamnesis and PE, patients who do not have clinical suspicion of COVID-19 and whose PCR result is negative, are planned to sign a specially prepared patient consent form for COVID-19 and undergo interventional treatment as soon as possible. For all patients who are planned to undergo anesthesia, it is also required to fill the COVID-19 Hospitalization Pre-Form (Form 1) prepared by the Scientific Committee of our hospital by the relevant services. We experienced that the use of this simple form, which was prepared in this period when our work intensity increased, was very useful in determining the details about patients that could be overlooked.

\section{Our Operational Order in the Operating Room for Patients with Confirmed or Suspected COVID-19 Planned for Surgical Intervention During the Pandemic Period}

During the pandemic, all patients are required to come to the operating room with a mask. The fever of the patients is measured again at the entrance of the operating room and they are accepted inside (1). In our operating room, one of the rooms at the closest possible distance to the entrance and exit has been determined as the COVID-19 room. In this room where patients with COVID-19 are taken, all materials and tools except the necessary materials are emptied. There is always a warning sign on the door of this room. Entries and exits to this room are prevented unless required during surgery. If a material outside the operation room is required, an outside staff brings it. The anesthesia machine, monitor, video laryngoscope, microscope and other space-occupying materials in the COVID-19 room where the patient will be operated are covered with transparent nylon bags to prevent the risk of contamination (Figure 3). Transparent covers are always kept backed up in an anesthesia emergency cabinet or in an accessible cabinet. Chief technicians and nurses in charge of the operating room are responsible for monitoring the PPE, transparent covers and protective measures to be used in the operating room.

Since the initial symptoms of COVID-19 infection are nonspecific and there is no high sensitivity test to rule out the presence of infection, personnel should wear PPE in all operations such as intubation/extubation/tracheotomy/tracheostomy, aspiration of respiratory secretions, mask-pouch ventilation, nasogastric catheter insertion/irrigation, cardiopulmonary resuscitation, bronchoscopy and endoscopy, which are known to cause aerosolization in the operating room $(1,2,9)$.

In order to put on and take off the PPE in the correct order, attention should be paid to the following steps:

Sequence for Putting on the PPE: All accessories except glasses are removed outside the operation room. Key, wallet, phone, etc. must be left out. Hair should be bulk. It should be remembered that the beard prevents the mask from fitting the face. Hand hygiene is provided after wearing disposable bonnets and foot protectors. Standard surgical hand washing/rubbing is performed after N95/FFP2 mask, goggles and face protector are worn. In the operating room, sterile surgical gowns and double layers of gloves are worn $(1,2)$.

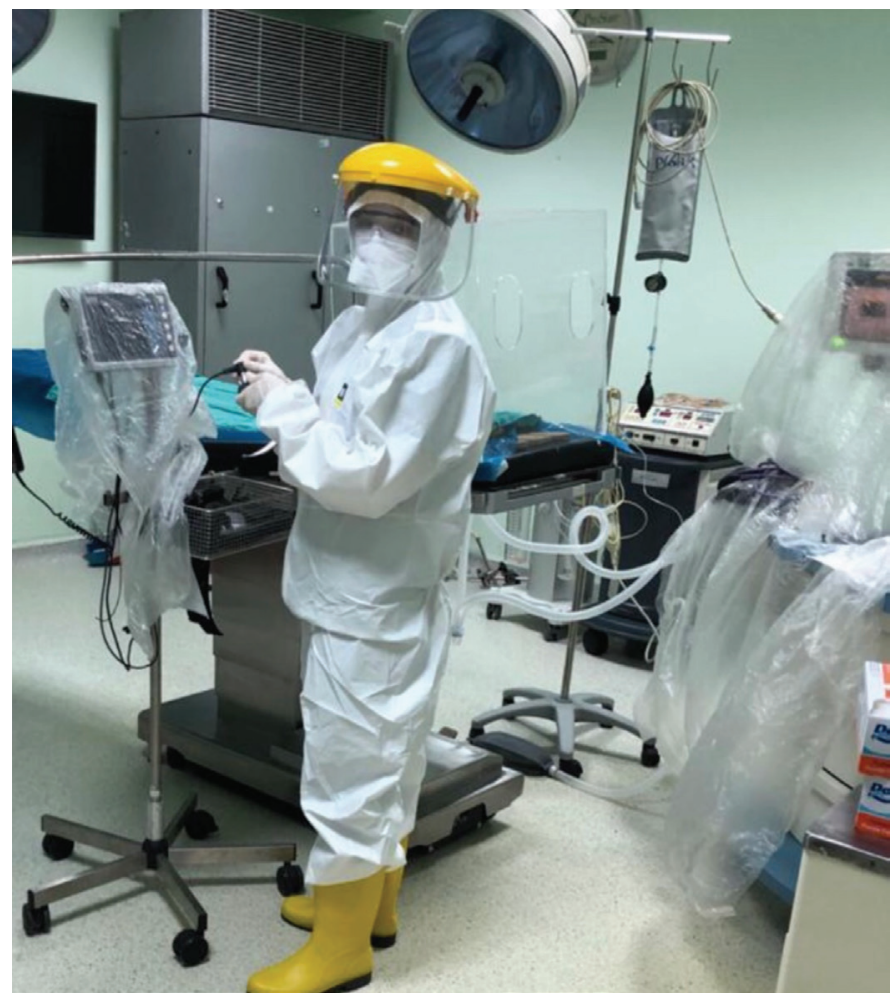

Figure 3. Protection of medical devices from contamination in operation rooms where patients with COVID-19 will be taken COVID-19: Coronavirus Disease-2019 


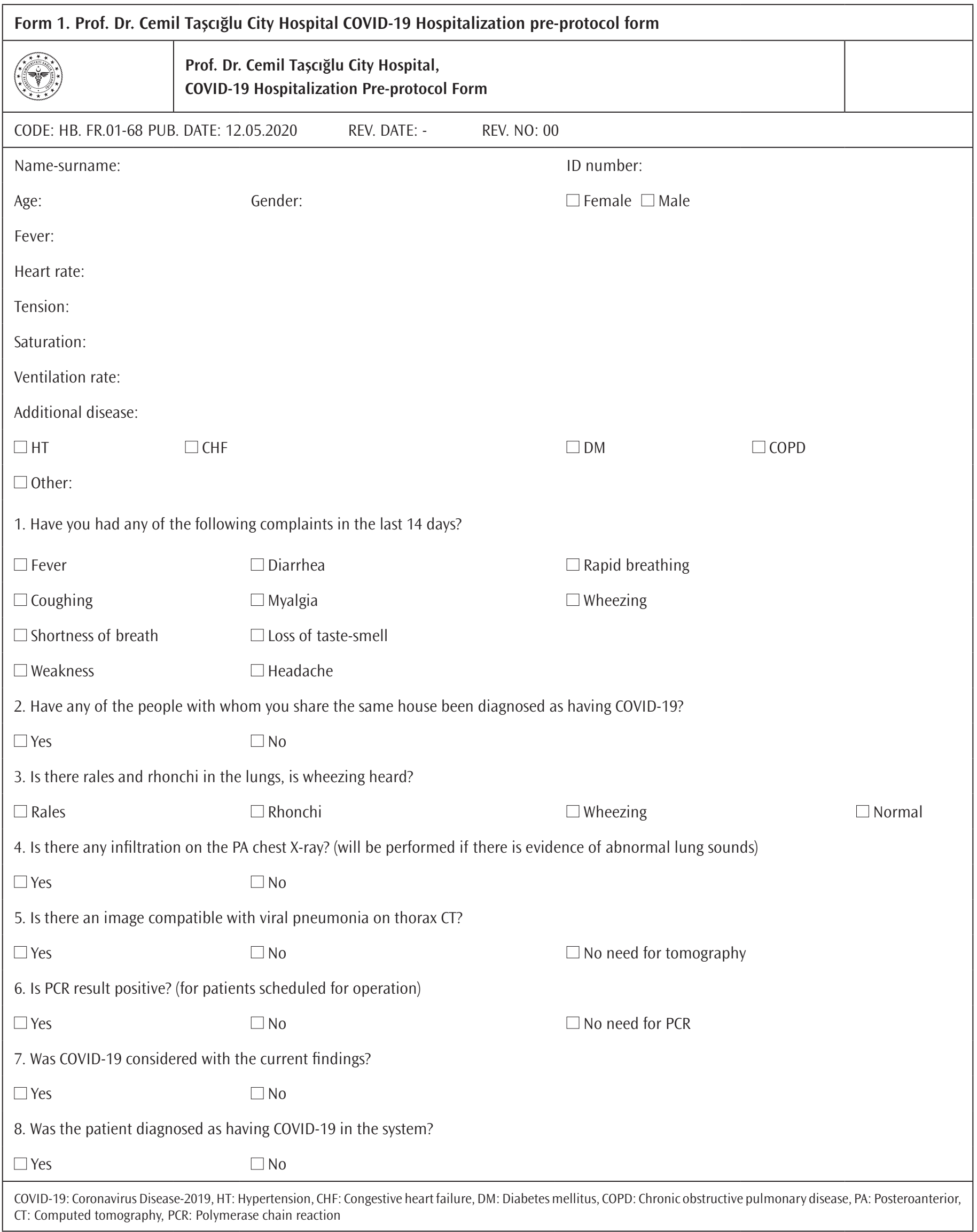


Sequence for Removing the PPE: In the operating room, hand hygiene is provided after removing galoshes, gloves and aprons. Outside the operation room, hand hygiene is provided after each of the glasses, face protector and bonnet is removed and then hand hygiene is provided again after the mask is removed. In the dressing room, the uniform is removed and a shower is taken if possible $(1,2,8)$. In particular, the last removal of the mask and providing hand hygiene after it should not be neglected. Non-disposable material (glasses and face protector) can be disinfected with $70 \%$ alcohol and reused. In the removal process, we should not be contacted with the dirty surface and hands should be washed with soap or a disinfectant should be used at every stage $(1,2,8)$.

Since intubation and extubation are among the procedures that create aerosolization, only the anesthesia team should be present in the room while performing these procedures, and healthcare workers who do not participate in the procedure should not be in the room $(1,2)$. The surgical team should enter the room 10 minutes after the procedure $(1,2)$. While the surgical team and operating room nurse leave the room before extubation, only the anesthesia team should stay in the room and extubation should be performed $(1,2)$.

If surgery can be performed with regional anesthesia, the first choice should be regional anesthesia. During regional anesthesia, oxygen should not be given with a mask, but if necessary oxygen should be given via nasal cannula, not more than $4 \mathrm{~mL} / \mathrm{min}$. Regional anesthesia and PPE should be worn in peripheral nerve blocks in patients with confirmed or suspected COVID-19 (9).

\section{Endotracheal Intubation}

In order to minimize aerosolization and droplet spread, intubation should be performed by the most experienced anesthesiologist and appropriate PPE should be worn. Awake fiberoptic intubation is avoided unless there is a specific indication. Rapid serial induction is recommended to avoid manual ventilation and aerosolization (9).

\section{Preoxygenation}

Preoxygenation can be performed with nasal oxygenation by giving $3 \mathrm{lt} / \mathrm{min} 100 \% \mathrm{O}_{2}$ under the mask during the apneic phase before intubation or by giving 5-8 breaths with $100 \% \mathrm{O}_{2}$ from the mask that is held with two hands under the transparent cover placed before induction. Endotracheal intubation should be administered as rapid serial intubation, and mask ventilation should be avoided as much as possible to prevent aerosolization. Cricoid pressure is not recommended in this period, intubation should be performed with a video laryngoscope at the first attempt, and a tube with appropriate size with a stylet should be ready (10). Oxygenation with low tidal volume/pressure is provided between unsuccessful attempts. If a straight tube is used, the endotracheal tube cuff is clamped so that the pilot is not damaged. The clamp should be opened and ventilation should be started only after the cuff is inflated and the system is switched to the closed system (10). It is important to inflate the cuff quickly and completely without any leakage. If intubation is unsuccessful for the second time, it is preferable to switch to supraglottic airway devices through which intubation can be performed. In the respiratory system, filters are attached at least to the expiration arm, preferably to both the inspiration and expiration arms and just after the intubation tube (10). If the breathing circuit is to disconnect from the tube, ventilation should be stopped first and the heat and moisture exchanger filter should be adjusted to remain at the patient side while the system is disconnected (10). There may be many barriers that can be used to prevent aerosolization during preoxygenation, intubation and ventilation. Transparent covers covered on the patient and intubation boxes called transparent 'aerosol boxes' (Figure 4) are among the most frequently used ones (11). It is important that the barrier used in these applications does not interfere with the interventions and that it is removed from the environment in a way that does not cause contamination after use (11).

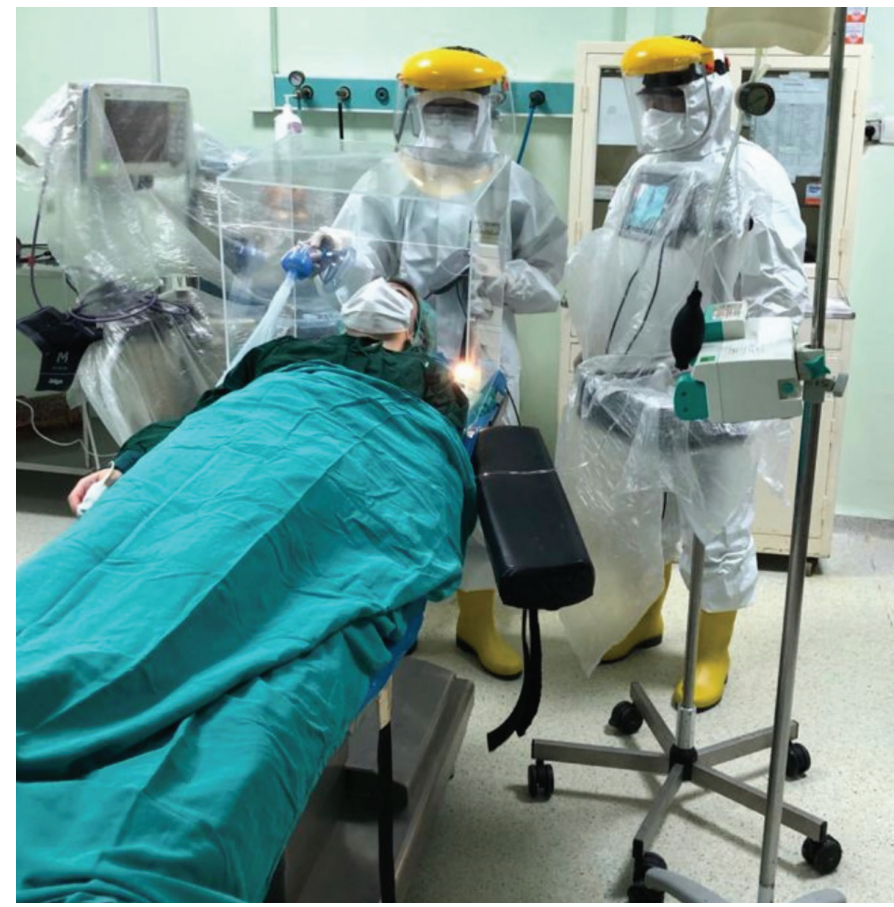

Figure 4. Transparent aerosol box used to prevent aerosolization in patients with COVID-19

COVID-19: Coronavirus Disease-2019 


\section{Extubation}

In order to restrict aerosolization during extubation, the mask can be taken under a transparent cover, and only the connector part can pierce the bag so that the mask remains under the cover. All aspirations of the patient are made with closed system aspiration, if possible. The transparent cover with the mask is brought closer to the patient's face and the patient is extubated. The intubation tube is thrown into the COVID-19 waste without contacting it.

If the patient is ventilated enough, the patient is ventilated with mask manipulations (such as head tilt - chin lift maneuver) without placing an airway in order to avoid coughing. If the patient needs respiratory support, he/she is ventilated with a circuit connected to the mask and with both hands. When the patient has sufficient vital capacity, an oxygen mask is put on his/her face. The patient, whose respiration is considered to be safe, is transferred from the operating room to the room in the service directly with an oxygen tube. All personnel who will participate in the transfer of patients with suspected or confirmed COVID-19 should wear PPE $(1,2)$. All patients with COVID-19 are transported with a special stretcher with a transparent tent barrier. Care should be taken to leave the stretcher ready for the next patient by wiping and cleaning it with 1/100 diluted bleach after each patient (1). After the patient leaves the room, the room and devices are cleaned. Video laryngoscope plates are cleaned with surface disinfectant cleanser and put into the box. Then, depending on the urgency, it is sterilized with savlon or surface disinfectant and used again. If it is to be disinfected in savlon, the area that needs to be closed in order not to damage the video laryngoscope blade is closed in accordance with the instructions and thrown into the savlon. In cases where the video laryngoscope is not used, surface disinfectant is sprayed on top of the materials such as laryngoscope blades and similar materials before they are being washed. Then, they are kept in a pre-prepared dirty box for at least two minutes and then sent to sterile processing department. In order to prevent contamination caused by dirty tools, boxes with lids which can be covered should be kept in every room, into which used dirty tools can be put and into which the chuck, mask, airway and blade of the laryngoscope can fit. The lids should be closed after spraying surface disinfectant on the materials placed in the dirty box, and diluted washing water or surface disinfectant should be sprayed on the box again $(1,2,9)$. Ensure that all contaminated equipment is placed in an appropriately labeled bin for support personnel who collect and dispose of the equipment.

\section{CONCLUSION}

As stated by the Scientific Advisory Board of the Ministry of Health in the "Working Guideline in Health Institutions During the Normalization Period" published in July 2020; due to the fact that the COVID-19 pandemic does not end completely, the risk continues until an effective treatment and/or vaccine specific to the virus is found. According to the same guide, it should be remembered that the flattening of the pandemic curve is not an indication that the total number of patients will decrease, it only indicates that the disease burden will spread over a longer period, and it is emphasized that health services should be initiated and maintained considering the continuity of the pandemic. It is important to make dynamic planning in order to manage the increasing demand due to the planned transactions delayed in this process. As long as the process continues, it is necessary to continue all protective measures, especially to prevent the loss of health employees' workforce.

\section{Ethics}

Peer-review: Externally and internally peer-reviewed.

\section{Authorship Contributions}

Surgical and Medical Practices: S.K., E.A.T., Concept: S.K., E.A.T., O.O., Design: S.K., E.A.T., O.O., T.M., I.G., Data Collection or Processing: S.K., E.A.T., O.O., I.G., Analysis or Interpretation: S.K., E.A.T., O.O., T.M., I.G., Literature Search: S.K., E.A.T., O.O., T.M., I.G., Writing: S.K., E.A.T., O.O., I.G.

Conflict of Interest: No conflict of interest was declared by the authors.

Financial Disclosure: The authors declared that this study received no financial support.

\section{REFERENCES}

1. T.C. Sağlık Bakanlığı Halk Sağ|ı̆ı Genel Müdürlüğü. Bilimsel Danışma Kurulu Çalışması, Covid-19 Rehberi; Enfeksiyon Kontrolü ve İzolasyon. Available from: URL: https://covid19.saglik.gov.tr/Eklenti/37699/0/ covid-19rehberienfeksiyonkontroluveizolasyonpdf.pdf

2. World Health Organisation. Rational use of personal protective equipment (PPE) for coronovirus disease (COVID-19): interim guidance. Available from: URL: https://apps.who.int/iris/handle/10665/331215

3. American College of Surgeons. Covid-19: Recommendations for Management of Elective Surgical Procedures. Available from: URL: https://www.facs.org/covid-19/clinical-guidance/elective-surgery

4. Royal College of Surgeons of England. Guidance For Surgeons Working During The Covid-19 Pandemic. Available from: URL: https://www. rcseng.ac.uk/coronavirus/joint-guidance-for-surgeons-v1/

5. American College of Surgeons. COVID-19: Elective Case Triage Guidelines for Surgical Care. Available from: URL: https://www.facs.org/covid-19/ clinical-guidance/elective-case 
6. American College of Surgeons. COVID-19: Guidance for Triage of NonEmergent Surgical Procedures. Available from: URL: https://www.facs. org/covid-19/clinical-guidance/triage

7. SAGES and EAES Recommendations Regarding Surgical Response to Covid-19 Crisis. Available from: URL: https://www.sages.org/ recommendations-surgical-response-covid-19/

8. T.C. Să̆glık Bakanlığı Bilimsel Danıșma Kurulu Çalıșması. Covid-19 Pandemisinde Normalleşme Döneminde Sağlık Kurumlarında Çalıșma Rehberi. Available from: URL: https://www.teb.org.tr/ versions_latest/1262/covid19-pandemisinde-normalle\%C5\%9Fmed\%C3\%B6neminde-sa\%C4\%9FI\%C4\%B1k-kurumlar\%C4\%B1nda\%C3\%87al\%C4\%B1\%C5\%9Fma-rehberi
9. Hacettepe Üniversitesi Erișkin ve Onkoloji Hastaneleri İnfeksiyon Kontrol Komitesi. Covid-19Kișisel Koruyucu Malzeme Kullanım Rehberi Available from: URL: http://www.hastane.hacettepe.edu.tr/pdf/ covid19_bilgilendirme_temmuz_2020.pdf

10. ASCA. Covid-19: American Society of Anesthesiologists Recommendations for Anesthesia Management Available from: URL: https://www. ascassociation.org/asca/resourcecenter/latestnewsresourcecenter/ covid-19/covid-19-anesthesia-recommendations

11. Canelli R, Connor CW, Gonzalez M, Nozari A, Ortega R. Barrier Enclosure during Endotracheal Intubation. N Engl J Med 2020;382:1957-8. 